\title{
Seroprevalence of hepatitis $A$ and $E$ virus infections among Allied Health Sciences students of the Faculty of Medicine, University of Jaffna
}

\author{
R Anusitha ${ }^{1}$, S Kajenthiny ${ }^{1}$, PAD Coonghe ${ }^{2}$, K Murugananthan $^{3}$
}

\section{Introduction}

Hepatitis A virus (HAV) and Hepatitis E virus (HEV) infections cause acute hepatitis, which remain as a major public health issue in developing countries. This study aimed to determine the seroprevalence of HAV and HEV infections in the Allied Health Sciences students of the Faculty of Medicine, University of Jaffna.

\section{Methods}

A descriptive cross-sectional study was conducted among 180 Allied Health Sciences students of the Faculty of Medicine, University of Jaffna. Blood samples were collected from June 2019 to July 2019 and a self-administered questionnaire was used to collect the data on risk factors for acquiring HAV and HEV infections. Serum samples were qualitatively analyzed for anti- HAV IgG and anti- HEV IgG using an ELISA kit (AccuDiag ${ }^{\mathrm{TM}}$, USA) and the results were reported as positive and negative.

\section{Results}

The study sample had 68 males and 112 females with a mean age of 23.5 years. The seroprevalence of HAV infection was found to be $99.4 \%$ and a seroprevalence of $0.6 \%$ was observed for the HEV infection.

\section{Conclusions}

This study suggests almost a 100 percent past exposure to HAV and it will be useful to investigate the number of participants that had hepatitis in the past and vaccination against HAV to identify the number that would have had IgG against HAV following hepatitis and vaccination.

Keywords: Hepatitis A virus infection, Hepatitis E virus infection, Seroprevalence, Risk factors

${ }^{1}$ Faculty of Allied Health Sciences, University of Jaffna, Sri Lanka

${ }^{2}$ Department of Community and Family Medicine, Faculty of Medicine, University of Jaffna, Sri Lanka 3Department of Microbiology, Faculty of Medicine, University of Jaffna, Sri Lanka 